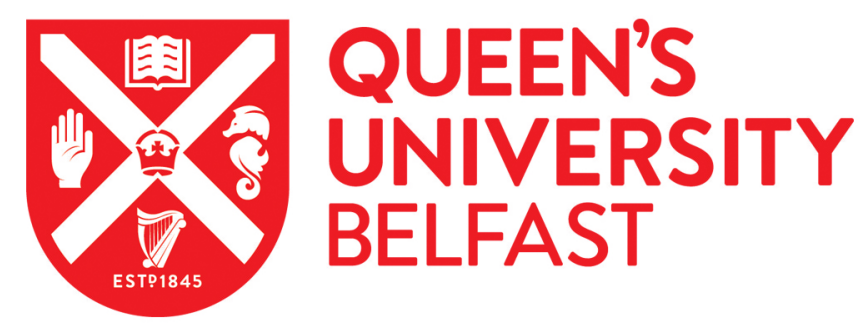

\title{
Secure Energy Harvesting Communications with Relay Selection over Nakagami-m Fading Channels
}

Yin, C., Nguyen, P., Garcia-Palacios, F., Tran, X., \& Le-Tien, T. (2017). Secure Energy Harvesting Communications with Relay Selection over Nakagami-m Fading Channels. Mobile Networks and Applications, 18. https://doi.org/10.1007/s11036-017-0919-5

\section{Published in:}

Mobile Networks and Applications

\section{Document Version:}

Peer reviewed version

Queen's University Belfast - Research Portal:

Link to publication record in Queen's University Belfast Research Portal

\section{Publisher rights}

Copyright 2017 Springer Verlag. This work is made available online in accordance with the publisher's policies. Please refer to any applicable terms of use of the publisher.

\section{General rights}

Copyright for the publications made accessible via the Queen's University Belfast Research Portal is retained by the author(s) and / or other copyright owners and it is a condition of accessing these publications that users recognise and abide by the legal requirements associated with these rights.

\section{Take down policy}

The Research Portal is Queen's institutional repository that provides access to Queen's research output. Every effort has been made to ensure that content in the Research Portal does not infringe any person's rights, or applicable UK laws. If you discover content in the Research Portal that you believe breaches copyright or violates any law, please contact openaccess@qub.ac.uk. 


\title{
Secure Energy Harvesting Communications with Relay Selection over Nakagami-m Fading Channels
}

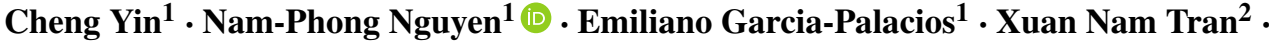 \\ Thuong Le-Tien ${ }^{3}$
}

(C) Springer Science+Business Media, LLC 2017

\begin{abstract}
In this paper, an energy harvesting relay network over Nakagami-m fading is investigated. In the considered system, the power beacon can provide wireless energy for the source and relays which deploy time-switching-based radio frequency energy harvesting technique. Two relay selection schemes, namely partial relay selection and optimal relay selection, are proposed in order to enhance the system performance. In the former, the source only has the channel state information of the first hop, while in the latter it has the full knowledge of the channel state information. The eavesdropper is able to wiretap to the signal transmitted from the source and the relays. The exact closed-form expressions of secrecy outage probability are derived. The results show that optimal relay selection performs better than partial relay selection. With increasing number of relays, the considered system shows better performance. In addition, the energy harvesting duration has a significant effect on the secrecy outage probability.
\end{abstract}

Keywords Physical layer security · Energy harvesting · Relay networks $\cdot$ Nakagami-m Fading

Nam-Phong Nguyen

pnguyen04@qub.ac.uk

1 Queen's University Belfast, Belfast, UK

2 Le Quy Don Technical University, Hanoi, Vietnam

3 Ho Chi Minh City University of Technology, Ho Chi Minh City, Vietnam

\section{Introduction}

Consuming energy in human's activities is one of the most significant reasons that lead to climate changes. Besides, the booming of wireless communication keeps increasing the number of mobile devices and base stations which cause a huge amount of carbon emission. Therefore, a more sustainable wireless network is one of the most urgent missions of wireless communication. Lately, radio frequency (RF) energy harvesting $(\mathrm{EH})$ has attracted wide attention from the research community as a promising solution [1-3]. This technology can enable mobile devices to harvest the RF signals in the surrounding environment and charge their batteries. It also allows energy constrained devices, i.e., wireless sensor nodes, prolong their lifetime while replacing or recharging their batteries is troublesome. In [4], the authors proposed theoretical power splitting schemes, i.e., time switching (TS), static power splitting (SPS), and on-off power splitting (OPS). The authors in [5] applied time switching-based relaying (TSR) protocol and power splitting-based relaying (PSR) protocol for relay networks. In [6], the application of TSR EH in a cooperative communication system was studied. In [7], the comparison between the best EH relay selection and the best information transmission relay selection was carried out. The authors in [8] examined a beamforming scheme in a multi-antenna fullduplex relay network. In [9], an EH relay system with multi-antenna power beacon was considered. The authors in [10] considered an EH system consists of multiple antenna BS, multiple single-antenna downlink and uplink users with the assumption that the uplink users have to harvest energy to transmit their information. A jointly beamforming and 
time allocating scheme that maximize the sum rate and energy efficiency of the considered system were proposed.

Enhancing the coverage of wireless networks is also an important aspect. Deploying higher transmit power at base stations can increase both the coverage and unwanted interference. The relay network is a solution for this problem since information can be transmitted from the transmitter to its destination with the help of one or some relay nodes. There are two well-known techniques deployed at relay nodes, i.e., amplify-and-forward (AF) and decodeand-forward (DF) [11]. In AF technique, the received signal at relays will be amplified and then transmitted to the next destination. Meanwhile, in DF technique, the signal is decoded first and then it is re-encoded before being transmitted to the next destination. In relay networks, sometimes, the link between the source and the relay node or the relay node to the destination experiences heavy fading or shadowing. One of the possible ways to enhance the performance of the system in this situation is to deploy multiple relay nodes and exploit the cooperative diversity of the network. Relay selection is a promising cooperative diversity method due to its low-complexity and high-efficiency. It has been proven that relay selection can offer full diversity order for AF and DF relay networks $[12,13]$.

One of the most significant problems of wireless networks is to secure information. Because of the broadcast nature of wireless networks, confidential information is vulnerable to malicious attacks. The traditional method to protect confidential information over wireless networks is to use upper layer cryptographic techniques. However, due to the rapid development in computing, traditional cryptographic techniques are no longer certain. It is urgent to develop new secure methods to aid the traditional ones. Recently, physical layer security (PLS) has gained widespread attention as an additional protecting layer of the wireless networks against eavesdroppers, alongside traditional cryptographic techniques. The principle of this technique is that the randomness of the wireless channels can be exploited to defend from eavesdroppers. The performance of PLS in relay networks has been well-investigated in the literature [14-26]. Recently, there have been studies on PLS for EH networks. In [27], the secrecy performance of EH system in which, wireless information and power are transferred concurrently was considered. A cooperative jamming scheme of an EH multi-antenna jammer was studied in [28]. In [20], the authors studied the secrecy performance of a wireless powered relay networks with aiding power beacon over Rayleigh fading channels. Nevertheless, the secrecy performance of EH relay networks has not been well investigated.

In this paper, the secrecy performance of a wireless powered relay networks with cooperating power beacon over Nakagami- $m$ fading channels is investigated.
The contributions of this paper are summarized as follows:

- Two relay selection protocols, i.e., optimal relay selection (ORS) and partial relay selection (PRS), are proposed to secure EH relay networks.

- The exact-closed form expressions of secrecy outage probability (SOP) for two relay selection protocols are derived.

- The results have shown that ORS scheme outperforms PRS scheme. Besides, increasing the number of relays significantly enhances the security performance of the considered system. In addition, the EH duration shows a huge effect on the SOP of the considered system and should be carefully designed.

The rest of the paper is organized as follows. System and channel models are described in Section 2. Performance analysis with exact closed-form expressions is developed in Section 3, while numerical results based on Monte-Carlo simulations to validate the correctness of our analyses are presented in section 4. Finally, the paper is concluded in Section 5.

\section{System and channel models}

We consider an energy harvesting network consisting of a power beacon $\mathrm{B}$, an information source $\mathrm{S}, K$ DF relays $\mathrm{R}_{k}, k=\{1, \ldots, K\}$, a destination $\mathrm{D}$ and an eavesdropper $\mathrm{E}$, as shown in Fig. 1. The power beacon B, S, $\mathrm{R}_{k}, \mathrm{E}$, and $D$ are equipped with single antenna. The additive white Gaussian noise (AWGN) at $\mathrm{R}_{k}$ and $\mathrm{D}$ has zero mean and variance $N_{0}$. Assuming all the channels are Nakagami-m fading, and the channel power gains $\left|h_{X}\right|^{2}$ are gamma distributed with mean power $\lambda_{X}$, and severity parameters $m_{X}$, where $X \in\{S R, S E, B S, B R, R E, R D\}$. The cumulative distribution function (CDF) and probability function (PDF) of $\left|h_{X}\right|^{2}$ can be written as [29]

$$
\begin{aligned}
& F_{X}(x)=1-\exp \left(-\frac{x}{\theta_{X}}\right) \sum_{i=0}^{m_{X}-1} \frac{1}{i !}\left(\frac{x}{\theta_{X}}\right)^{i}, \\
& f_{X}(x)=\frac{x^{m_{X}-1}}{\Gamma\left(m_{X}\right) \theta_{X}^{m_{X}}} \exp \left(-\frac{x}{\theta_{X}}\right) .
\end{aligned}
$$

Where $\theta_{X}=\frac{\lambda_{X}}{m_{X}}$, and $\Gamma(\cdot, \cdot)$ is the incomplete gamma function [30, Eq. (8.352.6)].

\subsection{Energy harvesting scheme}

In the proposed system, $\mathrm{S}$ and $\mathrm{R}_{k}$ can harvest energy from $\mathrm{B}$, and then transmit signals with the harvested energy. The power beacon can provide wireless energy to $\mathrm{S}$ and $\mathrm{R}_{k^{*}}$, 


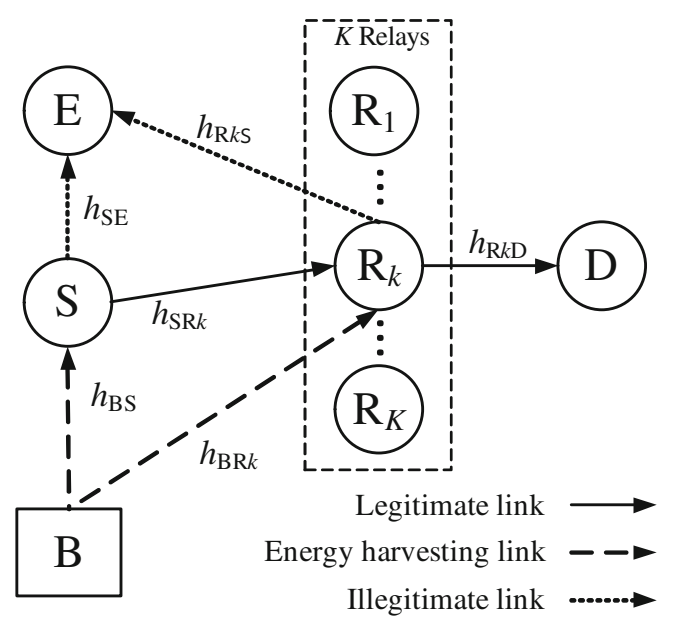

Fig. 1 System model

where the $\mathrm{R}_{k^{*}}$ is the selected aiding relay to forward information. In the paper, TS policy based energy harvesting is used, as shown in Fig. 2. In a transmission block time $T, \mathrm{~S}$ and $\mathrm{R}_{k^{*}}$ harvest energy for $\alpha T$ seconds. Then both $\mathrm{S} \rightarrow \mathrm{R}_{k^{*}}$ and $\mathrm{R}_{k^{*}} \rightarrow \mathrm{D}$ transmissions spend $(1-\alpha) T / 2$ equally to forward information, where $\alpha$ is the EH time fraction and $0<\alpha<1$. Therefore, the harvested energy at $\mathrm{S}$ and $\mathrm{R}_{k^{*}}$ are

$E_{\mathrm{S}}=\eta \mathcal{P}_{\mathrm{B}} \alpha T\left|h_{\mathrm{BS}}\right|^{2}$,

$E_{\mathrm{R}}=\eta \mathcal{P}_{\mathrm{B}} \alpha T\left|h_{\mathrm{BR}_{k^{*}}}\right|^{2}$,

where $\eta$ is the efficiency coefficient and $0<\eta<1$. $\mathcal{P}_{\mathrm{B}}$ is the transmit power of power beacon B. $\left|h_{\mathrm{BS}}\right|^{2}$ and $\left|h_{\mathrm{BR}_{k^{*}}}\right|^{2}$ are channel links from power beacon $B$ to source $S$ and $B$ to selected aiding relay $R_{k^{*}}$. Assuming the processing energy at $S$ and $R_{k^{*}}$ can be ignored. Therefore, the transmit power of $S$ and $\mathrm{R}_{k^{*}}$ are

$$
\begin{aligned}
& \mathcal{P}_{\mathrm{S}}=\frac{2 \eta \mathcal{P}_{\mathrm{B}}\left|h_{\mathrm{BS}}\right|^{2} \alpha}{(1-\alpha)}, \\
& \mathcal{P}_{\mathrm{R}}=\frac{2 \eta \mathcal{P}_{\mathrm{B}}\left|h_{\mathrm{BR}_{k^{*}}}\right|^{2} \alpha}{(1-\alpha)} .
\end{aligned}
$$

\subsection{Security scenarios}

In the considered system, $E$ can eavesdrop information during the $S \rightarrow R_{k^{*}}$ and $R_{k^{*}} \rightarrow D$ transmissions. We assume that there is no direct link from $B \rightarrow E$. Therefore, the harvested energy at $S$ and $\mathrm{R}_{k^{*}}$ can not be disturbed in the presence of E. DF technique and different code books are used in order to enhance the performance of the considered system. The secrecy capacity of the considered system is written as

$C_{s}=\min \left(C_{1 s}, C_{2 s}\right)$,

where $C_{1 s}$ and $C_{2 s}$ are the achievable secrecy rate of the first hop and the second hop, they can be expressed as follows:

$$
\begin{aligned}
C_{1 s} & =\frac{1-\alpha}{2}\left[\log _{2}\left(\frac{1+\gamma_{1 \mathrm{M}}}{1+\gamma_{1 \mathrm{E}}}\right)\right]^{+} \\
& =\epsilon\left[\log _{2}\left(\frac{1+\gamma_{1 \mathrm{M}}}{1+\gamma_{1 \mathrm{E}}}\right)\right]^{+}, \\
C_{2 s} & =\frac{1-\alpha}{2}\left[\log _{2}\left(\frac{1+\gamma_{2 \mathrm{M}}}{1+\gamma_{2 \mathrm{E}}}\right)\right]^{+} \\
& =\epsilon\left[\log _{2}\left(\frac{1+\gamma_{2 \mathrm{M}}}{1+\gamma_{2 \mathrm{E}}}\right)\right]^{+},
\end{aligned}
$$

where $\epsilon=\frac{1-\alpha}{2}$ accounts for the fact that during a block time $T$, both the first hop and second hop spend $(1-\alpha) T / 2$ equally to forward information, and $[x]^{+}=\max (x, 0) \cdot \gamma_{1 \mathrm{M}}$ is the signal-to-noise-ratio (SNR) of the first link $\mathrm{S} \rightarrow \mathrm{R}_{k^{*}}$, $\gamma_{2 \mathrm{M}}$ is the SNR of the second link $\mathrm{R}_{k^{*}} \rightarrow \mathrm{D}, \gamma_{1 \mathrm{E}}$ is the SNR at $S \rightarrow E$ and $\gamma_{2 E}$ is the SNR at $R_{k^{*}} \rightarrow E$. The SNR of the first hop $\gamma_{1 \mathrm{M}}$ is given as

$$
\begin{aligned}
\gamma_{1 \mathrm{M}} & =\frac{\mathcal{P}_{\mathrm{S}}\left|h_{\mathrm{SR}_{k^{*}}}\right|^{2}}{N_{0}} \\
& =\frac{2 \eta \alpha \mathcal{P}_{\mathrm{B}}\left|h_{\mathrm{BS}}\right|^{2}\left|h_{\mathrm{SR}_{k^{*}}}\right|^{2}}{N_{0}(1-\alpha)} \\
& =\xi \gamma_{\mathrm{M}}\left|h_{\mathrm{BS}}\right|^{2}\left|h_{\mathrm{SR}_{k^{*}}}\right|^{2},
\end{aligned}
$$

where $\gamma_{\mathrm{M}}=\frac{\mathcal{P}_{\mathrm{B}}}{N_{0}}, \xi=\frac{2 \eta \alpha}{(1-\alpha)}$, and $\left|h_{\mathrm{SR}_{k^{*}}}\right|^{2}$ is the channel power gain of $S \rightarrow R_{k^{*}}$ link. Similarly, $\gamma_{2 \mathrm{M}}, \gamma_{1 \mathrm{E}}$, and $\gamma_{2 \mathrm{E}}$ can be written as

$$
\begin{aligned}
& \gamma_{2 \mathrm{M}}=\gamma_{\mathrm{M}} \xi\left|h_{\mathrm{BR}_{k^{*}}}\right|^{2}\left|h_{\mathrm{R}_{k^{*}} \mathrm{D}}\right|^{2}, \\
& \gamma_{1 \mathrm{E}}=\gamma_{\mathrm{E}} \xi\left|h_{\mathrm{BS}}\right|^{2}\left|h_{\mathrm{SE}}\right|^{2}, \\
& \gamma_{2 \mathrm{E}}=\gamma_{\mathrm{E}} \xi\left|h_{\mathrm{BR}_{k}}\right|^{2}\left|h_{\mathrm{R}_{k^{*}}}\right|^{2},
\end{aligned}
$$

where $\left|h_{\mathrm{R}_{k^{*}} \mathrm{D}}\right|^{2},\left|h_{\mathrm{SE}}\right|^{2}$, and $\left|h_{\mathrm{R}_{k^{*}} \mathrm{E}}\right|^{2}$ are the channel power gains of $\mathrm{R}_{k^{*}} \rightarrow \mathrm{D}, \mathrm{S} \rightarrow \mathrm{E}$, and $\mathrm{R}_{k^{*}} \rightarrow \mathrm{E}$ links. $\gamma_{\mathrm{E}}=\frac{\mathcal{P}_{\mathrm{B}}}{N_{\mathrm{E}}}$, and $N_{\mathrm{E}}$ is the variance of the AWGN at E.

\subsubsection{Partial relay selection (PRS)}

In some networks, it is not possible to know the full knowledge of channel state information (CSI) of all the links. Therefore, in this paper, we first consider a PRS that only
Fig. 2 Time switching based protocol

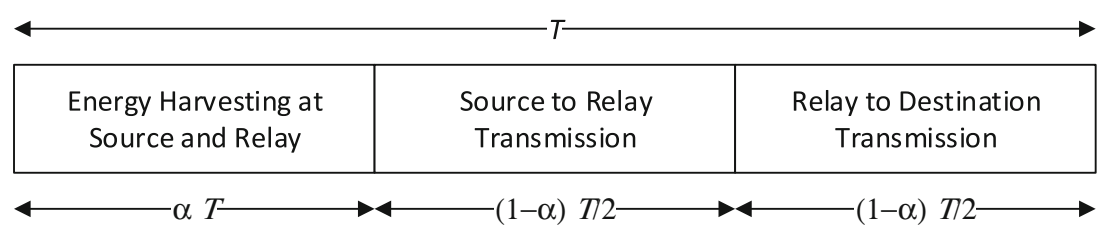


need to know the CSI of the first hop $\mathrm{S} \rightarrow \mathrm{R}_{k}$. The best link is selected when the first hop has the maximum SNR, and can be expressed as

$k^{*}=\arg \max _{k=1, \ldots, K}\left(\left|h_{\mathrm{SR}_{k}}\right|^{2}\right)$.

Therefore, according to Eq. 14, the SNR of the first hop, given by Eq. 10, can be rewritten as

$\gamma_{1 \mathrm{M}}=\gamma_{\mathrm{M}} \xi\left|h_{\mathrm{BS}}\right|^{2} \max _{k=1, \ldots, K}\left(\left|h_{\mathrm{SR}_{k^{*}}}\right|^{2}\right)$.

The achievable secrecy rates of PRS scheme in the considered system is written as

$$
\begin{aligned}
C_{\mathrm{PRS}}= & \epsilon\left[\operatorname { l o g } _ { 2 } \operatorname { m i n } \left(\frac{1+\gamma_{\mathrm{M}} \xi\left|h_{\mathrm{BS}}\right|^{2}\left|h_{\mathrm{SR}_{k^{*}}}\right|^{2}}{1+\gamma_{\mathrm{E}} \xi\left|h_{\mathrm{BS}}\right|^{2}\left|h_{\mathrm{SE}}\right|^{2}},\right.\right. \\
& \left.\left.\frac{1+\gamma_{\mathrm{M}} \xi\left|h_{\mathrm{BR}_{k^{*}}}\right|^{2}\left|h_{\mathrm{R}_{k^{*}} \mathrm{D}}\right|^{2}}{1+\gamma_{\mathrm{E}} \xi\left|h_{\mathrm{BR}_{k^{*}}}\right|^{2}\left|h_{\mathrm{R}_{k^{*}} \mathrm{E}}\right|^{2}}\right)\right]^{+} .
\end{aligned}
$$

\subsubsection{Optimal relay selection (ORS)}

Unlike PRS that only has part of CSI of the considered system, in ORS scheme, we assume that $S$ has full knowledge of CSI of all the links. The aiding relay $\mathrm{R}_{k^{*}}$ is chosen that can help the system have the maximum secrecy capacity, and can be expressed as

$$
\begin{aligned}
k^{*}= & \arg \max _{k=1, \ldots, K}\left[\operatorname { m i n } \left(\frac{1+\gamma_{\mathrm{M}} \xi\left|h_{\mathrm{BS}}\right|^{2}\left|h_{\mathrm{SR}_{k}}\right|^{2}}{1+\gamma_{\mathrm{E}} \xi\left|h_{\mathrm{BS}}\right|^{2}\left|h_{\mathrm{SE}}\right|^{2}},\right.\right. \\
& \left.\left.\frac{1+\gamma_{\mathrm{M}} \xi\left|h_{\mathrm{BR}_{k}}\right|^{2}\left|h_{\mathrm{R}_{k} \mathrm{D}}\right|^{2}}{1+\gamma_{\mathrm{E}} \xi\left|h_{\mathrm{BR}_{k}}\right|^{2}\left|h_{\mathrm{R}_{k} \mathrm{E}}\right|^{2}}\right)\right] .
\end{aligned}
$$

The achievable secrecy rates of ORS scheme in the considered system is written as

$$
\begin{aligned}
& C_{\mathrm{ORS}}=\epsilon\left[\operatorname { l o g } _ { 2 } \operatorname { m a x } _ { k = 1 , \ldots , K } \left[\operatorname { m i n } \left(\frac{1+\gamma_{\mathrm{M}} \xi\left|h_{\mathrm{BS}}\right|^{2}\left|h_{\mathrm{SR}_{k}}\right|^{2}}{1+\gamma_{\mathrm{E}} \xi\left|h_{\mathrm{BS}}\right|^{2}\left|h_{\mathrm{SE}}\right|^{2}},\right.\right.\right. \\
& \left.\left.\left.\frac{1+\gamma_{\mathrm{M}} \xi\left|h_{\mathrm{BR}_{k}}\right|^{2}\left|h_{\mathrm{R}_{k} \mathrm{D}}\right|^{2}}{1+\gamma_{\mathrm{E}} \xi\left|h_{\mathrm{BR}_{k}}\right|^{2}\left|h_{\mathrm{R}_{k} \mathrm{E}}\right|^{2}}\right)\right]\right]^{+} \text {. }
\end{aligned}
$$

\section{Secrecy outage probability}

In this section, the closed-form expressions of secrecy outage probability (SOP) for PRS and ORS schemes are provided in order to evaluate the security performance of the considered system. In a communication system, the security outage probability is defined by the probability that the achievable secrecy rate is below a pre-defined threshold $R_{t h}$, and can be expressed as

$$
\begin{aligned}
\mathbb{P}\left\{C<R_{t h}\right\} & =\mathbb{P}\{\gamma<\beta\} \\
& =F_{\gamma \mathrm{PPS}}(\beta),
\end{aligned}
$$

where $C=\log _{2}(1+\gamma), \gamma$ is the SNR of the considered system, and $\beta=2^{\frac{R_{t h}}{\epsilon}}$.

\subsection{Partial relay selection}

From Eqs. 16 and 19, we have

$\mathbb{P}\left\{C_{\mathrm{PRS}}<R_{t h}\right\}=\mathbb{P}\left\{\gamma_{\mathrm{PRS}}<\beta\right\}$

$$
=F_{\gamma_{\mathrm{PRS}}}(\beta) \text {, }
$$

where

$$
\begin{aligned}
\gamma_{\mathrm{PRS}}=\min \left(\frac{1+\gamma_{\mathrm{M}} \xi\left|h_{\mathrm{BS}}\right|^{2}\left|h_{\mathrm{SR}_{k^{*}}}\right|^{2}}{1+\gamma_{\mathrm{E}} \xi\left|h_{\mathrm{BS}}\right|^{2}\left|h_{\mathrm{SE}}\right|^{2}},\right. \\
\left.\frac{1+\gamma_{\mathrm{M}} \xi\left|h_{\mathrm{BR}_{k^{*}}}\right|^{2}\left|h_{\mathrm{R}_{k^{*}} \mathrm{D}}\right|^{2}}{1+\gamma_{\mathrm{E}} \xi\left|h_{\mathrm{BR}_{k^{*}}}\right|^{2}\left|h_{\mathrm{R}_{k^{*}}}\right|^{2}}\right) .
\end{aligned}
$$

$F_{\gamma_{\text {PRS }}}(\beta)$ is the CDF of $\gamma_{\text {PRS }}$. From Eq. 20 , we have the following Lemma.

Lemma 1 The SOP of the considered system in PRS scheme is formulated as follows:

$$
\begin{aligned}
& F_{\gamma_{\mathrm{PRS}}}(\beta)=1+\sum_{k=1}^{k} \sum_{v=0}^{l} \sum_{l=0}^{k\left(m_{\mathrm{SR}}-1\right)} \sum_{i=0}^{m_{\mathrm{RD}}-1} \sum_{j=0}^{i}\left(\begin{array}{l}
k \\
k
\end{array}\right)\left(\begin{array}{l}
l \\
v
\end{array}\right)\left(\begin{array}{l}
i \\
j
\end{array}\right)(-1)^{k}(\beta-1)^{l-v+i-j}\left(\gamma_{\mathrm{E}} \beta\right)^{v+j} \\
& \times \frac{w\left(l, k, m_{\mathrm{SR}}\right)}{i ! \gamma_{\mathrm{M}}^{l+i} \xi{ }^{l-v+i-j} \theta_{\mathrm{SR}}^{l} \theta_{\mathrm{RD}}^{i} \Gamma\left(m_{\mathrm{SE}}\right) \theta_{\mathrm{SE}}^{m_{\mathrm{SE}}} \Gamma\left(m_{\mathrm{BS}}\right) \theta_{\mathrm{BS}}^{m_{\mathrm{BS}}} \Gamma\left(m_{\mathrm{RE}}\right) \theta_{\mathrm{RE}}^{m_{\mathrm{RE}}} \Gamma\left(m_{\mathrm{BR}}\right) \theta_{\mathrm{BR}}^{m_{\mathrm{BR}}}} \\
& \times \Gamma\left(v+m_{\mathrm{SE}}\right)\left(\frac{k \beta}{\theta_{\mathrm{SR}}}+\frac{1}{\theta_{\mathrm{SE}}}\right)^{-\left(v+m_{\mathrm{SE}}\right)} \Gamma\left(v+m_{\mathrm{RE}}\right)\left(\frac{\beta \gamma_{\mathrm{E}}}{\gamma_{\mathrm{M}} \theta_{\mathrm{RD}}}+\frac{1}{\theta_{\mathrm{RE}}}\right)^{-\left(j+m_{\mathrm{RE}}\right)} \\
& \times 2 \times\left(\frac{k \theta_{\mathrm{BS}}(\beta-1)}{\theta_{\mathrm{SR}} \gamma_{\mathrm{M}} \xi}\right)^{\frac{m_{\mathrm{BS}}-l+v}{2}} \times 2 \times\left(\frac{\theta_{\mathrm{BR}}(\beta-1)}{\theta_{\mathrm{RD}} \gamma_{\mathrm{M}} \xi}\right)^{\frac{m_{\mathrm{BR}}-i+j}{2}} \mathbf{K}_{m_{\mathrm{BS}}-l+v}\left(2 \sqrt{\frac{k(\beta-1)}{\theta_{\mathrm{SR}} \theta_{\mathrm{BS}} \gamma_{\mathrm{M}} \xi}}\right) \\
& \times \mathbf{K}_{m_{\mathrm{BR}}-i+j}\left(2 \sqrt{\frac{\beta-1}{\theta_{\mathrm{RD}} \theta_{\mathrm{BR}} \gamma_{\mathrm{M}} \xi}}\right) .
\end{aligned}
$$


where $\mathbf{K}_{1}(\cdot)$ defined in [30, Eq. (3.471.9)] is the modified Bessel function of the second kind and $w(\cdot, \cdot, \cdot)$ function is derived as

$w\left(l, k, m_{\mathrm{SR}}\right)=\left\{\begin{array}{ll}\left(\frac{1}{l !}\right)^{k}, & \text { if } l=0 \\ \frac{k}{l !}, & \text { if } l=1 \\ \frac{1}{l} \sum_{q=1}^{l}(q k-l+q) \frac{1}{q !} w\left(l-q, k, m_{\mathrm{SR}}\right), & \text { if } 2 \leqslant l \leqslant\left(m_{\mathrm{SR}}-1\right) \\ \frac{1}{l} \sum_{q=1}^{m_{\mathrm{SR}}-1}(q k-l+q) \frac{1}{q !} w\left(l-q, k, m_{\mathrm{SR}}\right), & \text { if } m_{\mathrm{SR}} \leqslant l<k\left(m_{\mathrm{SR}}-1\right)\end{array}\right.$.

Proof The proof is given in Appendix A.

\subsection{Optimal relay selection}

From Eqs. 18 and 19, we have

$$
\begin{aligned}
\mathbb{P}\left\{C_{\text {ORS }}<R_{t h}\right\} & =\mathbb{P}\left\{\gamma_{\text {ORS }}<\beta\right\} \\
& =F_{\gamma_{\text {ORS }}}(\beta) .
\end{aligned}
$$

where

$$
\begin{aligned}
\gamma_{\mathrm{ORS}}= & \max _{k=1, \ldots, K}\left[\operatorname { m i n } \left(\frac{1+\gamma_{\mathrm{M}} \xi\left|h_{\mathrm{BS}}\right|^{2}\left|h_{\mathrm{SR}_{k}}\right|^{2}}{1+\gamma_{\mathrm{E}} \xi\left|h_{\mathrm{BS}}\right|^{2}\left|h_{\mathrm{SE}}\right|^{2}},\right.\right. \\
& \left.\left.\frac{1+\gamma_{\mathrm{M}} \xi\left|h_{\mathrm{BR}_{k}}\right|^{2}\left|h_{\mathrm{R}_{k} \mathrm{D}}\right|^{2}}{1+\gamma_{\mathrm{E}} \xi\left|h_{\mathrm{BR}_{k}}\right|^{2}\left|h_{\mathrm{R}_{k} \mathrm{E}}\right|^{2}}\right)\right]
\end{aligned}
$$

$F_{\gamma_{\text {ORS }}}(\beta)$ is the CDF of $\gamma_{\text {ORs. }}$. From Eq. 18 , we have the following Lemma.
Lemma 2 The SOP of optimal relay selection in the considered system is calculated as follows:

$$
\begin{aligned}
& F_{\gamma_{\mathrm{ORS}}}(\beta) \\
& =1+\sum_{k=1}^{K}\left(\begin{array}{l}
K \\
k
\end{array}\right)(-1)^{k} \Theta \sum_{i=0}^{k\left(m_{\mathrm{SR}}-1\right)} w\left(i, k, m_{\mathrm{SR}}\right) \\
& \times \sum_{n=0}^{i}\left(\begin{array}{l}
i \\
n
\end{array}\right)\left(\frac{\beta-1}{\gamma_{\mathrm{M}} \xi \theta_{\mathrm{SR}}}\right)^{i-n}\left(\frac{\gamma_{\mathrm{E}} \beta}{\gamma_{\mathrm{M}} \theta_{\mathrm{SR}}}\right)^{n} \\
& \times 2 \times\left(\frac{\theta_{\mathrm{BS}} k(\beta-1)}{\gamma_{\mathrm{M}} \xi \theta_{\mathrm{SR}}}\right)^{\frac{n-i+m_{\mathrm{BS}}}{2}} \\
& \times \mathbf{K}_{n-i+m_{\mathrm{BS}}}\left(2 \sqrt{\frac{k(\beta-1)}{\gamma_{\mathrm{M}} \xi \theta_{\mathrm{SR}} \theta_{\mathrm{BS}}}}\right) \frac{\Gamma\left(n+m_{\mathrm{SE}}\right)}{\left(\frac{1}{\theta_{\mathrm{SE}}}+\frac{\gamma_{\mathrm{E}} \beta k}{\gamma_{\mathrm{M}} \theta_{\mathrm{SR}}}\right)^{n+m_{\mathrm{SE}}}} \\
& \times \frac{1}{\Gamma\left(m_{\mathrm{BS}}\right) \theta_{\mathrm{BS}}^{m_{\mathrm{BS}}} \Gamma\left(m_{\mathrm{SE}}\right) \theta_{\mathrm{SE}}^{m_{\mathrm{SE}}}} .
\end{aligned}
$$

Where

$$
\begin{aligned}
& \Theta=\left[\sum_{u=0}^{m_{\mathrm{RD}}-1} \sum_{v=0}^{u}\left(\begin{array}{l}
u \\
v
\end{array}\right) \frac{1}{u !} \frac{(\beta-1)^{u-v}\left(\gamma_{\mathrm{E}} \beta\right)^{v}}{\gamma_{\mathrm{M}}^{u} \xi^{u-v} \theta_{\mathrm{RD}}^{u}} \frac{1}{\Gamma\left(m_{\mathrm{RE}}\right) \theta_{\mathrm{RE}}^{m_{\mathrm{RE}}}} \frac{1}{\Gamma\left(m_{\mathrm{BR}}\right) \theta_{\mathrm{BR}}^{m_{\mathrm{BR}}}}\right. \\
& \times \Gamma\left(v+m_{\mathrm{RE}}\right)\left(\frac{\gamma_{\mathrm{E}} \beta}{\gamma_{\mathrm{M}} \theta_{\mathrm{RD}}}+\frac{1}{\theta_{\mathrm{RE}}}\right)^{-\left(v+m_{\mathrm{RE}}\right)} 2 \times\left(\frac{[\beta-1] \theta_{\mathrm{BR}}}{\gamma_{\mathrm{M}} \xi \theta_{\mathrm{RD}}}\right)^{\frac{v-u+m_{\mathrm{BR}}}{2}} \\
& \left.\times \mathbf{K}_{v-u+m_{\mathrm{BR}}}\left(2 \sqrt{\frac{\beta-1}{\gamma_{\mathrm{M}} \xi \theta_{\mathrm{RD}} \theta_{\mathrm{BR}}}}\right)\right]^{k}
\end{aligned}
$$

and

$$
w\left(i, k, m_{\mathrm{SR}}\right)= \begin{cases}1, & \text { if } i=0 \\ k, & \text { if } i=1 \\ \left(\frac{1}{\left(m_{\mathrm{SR}}-1\right) !}\right)^{k}, & \text { if } i=k\left(m_{\mathrm{SR}}-1\right) \\ \frac{1}{i} \sum_{t=1}^{i}(t k-i+t) \frac{1}{t !} w\left(i-t, m_{\mathrm{SR}}, k\right), & \text { if } 2 \leqslant i \leqslant m_{\mathrm{SR}}-1 \\ \frac{1}{i} \sum_{t=1}^{m_{\mathrm{SR}}-1}(t k-i+t) \frac{1}{t !} w\left(i-t, m_{\mathrm{SR}}, k\right), & \text { if } m_{\mathrm{SR}} \leqslant i \leqslant k\left(m_{\mathrm{SR}}-1\right) .\end{cases}
$$


Proof The proof is given in Appendix B.

\section{Numerical results}

In this section, the simulation results using Monte Carlo method are provided to validate the accuracy of the above secrecy outage probability analysis. In this section, the following parameters are fixed: $\gamma_{\mathrm{E}}=10 \mathrm{~dB}, \mathrm{R} t h=0.2 \mathrm{bits} / \mathrm{s} / \mathrm{Hz}$, $\eta=0.8$. The parameters of channel links are fixed: $m_{\mathrm{BS}}=2$, $\theta_{\mathrm{BS}}=10, m_{\mathrm{BR}}=2, \theta_{\mathrm{BR}}=10, m_{\mathrm{SR}}=2, \theta_{\mathrm{SR}}=10, a=2, \theta_{\mathrm{RD}}=10$. The parameters of the eavesdropping links are designed: $m_{\mathrm{SE}}=2, \theta_{\mathrm{SE}}=2, m_{\mathrm{RE}}=2, \theta_{\mathrm{RE}}=2$.

Figure 3 shows the influence of the number of relays on the secrecy outage probability of PRS and ORS schemes. In this setup, $\alpha=0.01$. When $K$ increases, the SOP of the considered system decreases. ORS has better performance than PRS scheme because it has more knowledge of CSI of the considered system than PRS. Besides, the number of relays has more effect on ORS than on PRS. In fixed $\gamma_{M}$, ORS benefits from increasing $K$ more than PRS. In addition, in the high $\gamma_{\mathrm{M}}$ region, the SOP for different multiple number of relays shows little difference compared to PRS scheme.

Figure 4 investigates the effect of energy harvesting time in a transmission block time on the secrecy outage probability. In the considered system, $k=3, \gamma_{\mathrm{M}}=10 \mathrm{~dB}$. In both PRS and ORS schemes, the SOP is very large when $\alpha$ is too small or too high. This is because when the energy harvesting time is too long, $\mathrm{S}$ and $\mathrm{R}_{k^{*}}$ do not have sufficient time to forward information and when the transmission duration is too long, there is little time for the nodes to harvest energy. In both cases, the SOP will be extremely large. The results

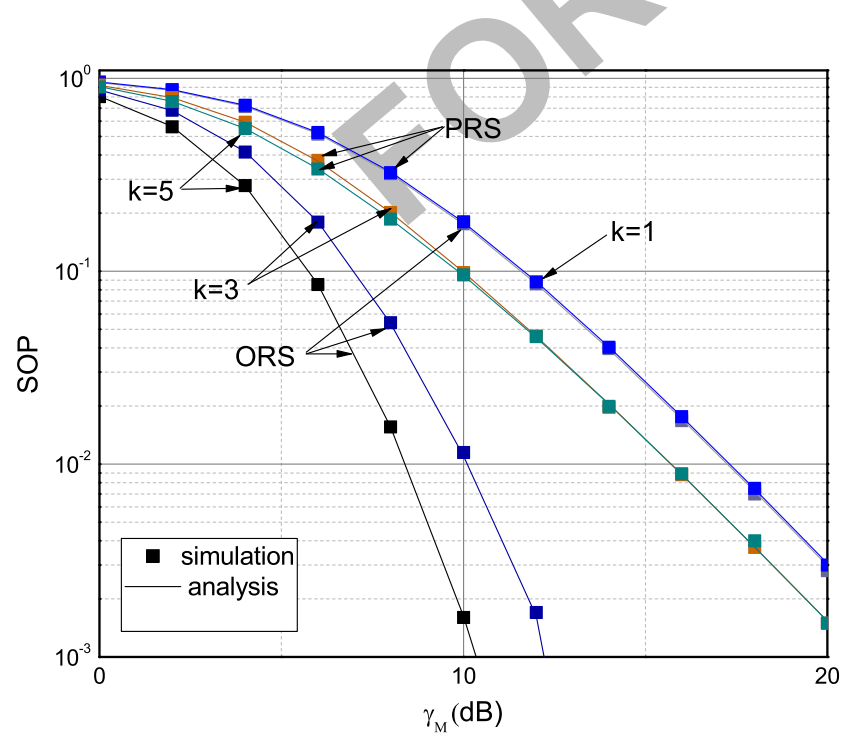

Fig. 3 SOP in ORS and PRS schemes with different number of relays

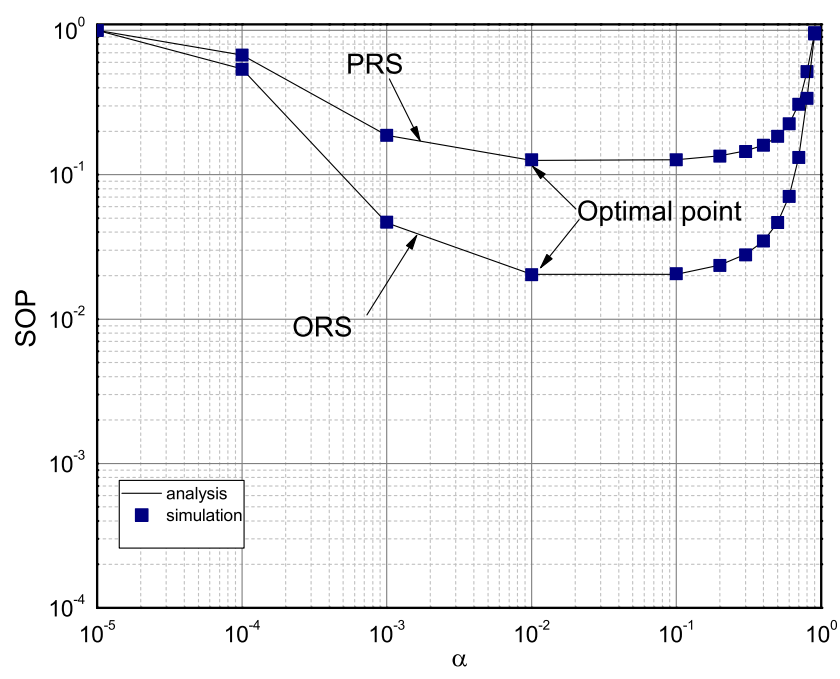

Fig. 4 SOP in ORS and PRS schemes is plotted as a function of $\alpha$

also show that the SOP has an optimal point at $\alpha=10^{-2}$ in PRS and ORS schemes. Specifically, there is very little difference in the SOP when $\alpha$ is between $10^{-2}$ and $10^{-1}$. In general, $\alpha$ has a significant effect on the performance of the considered system. Therefore, the EH duration should be mindfully determined in order to secure the communication.

\section{Conclusions}

In this paper, a system with multiple DF relays and a power beacon with single antenna in the presence of an eavesdropper has been investigated. Two relay selection schemes have been proposed in order to enhance the performance of the considered system. The exact closed-form expressions of SOP have been derived. The results show that ORS scheme has better performance than PRS. With the increasing number of relays, the security performance can be enhanced. Finally, the EH duration has huge impact on the system performance and should be carefully designed. In our examples, there is an optimal point at $\alpha=10^{-2}$.

\section{Appendix A: proof of lemma 1}

The SNR of first hop and second hop in PRS scheme can be written as

$$
\begin{aligned}
& \gamma_{1 \mathrm{PRS}}=\frac{1+\gamma_{\mathrm{M}} \xi\left|h_{\mathrm{BS}}\right|^{2}\left|h_{\mathrm{SR}_{k^{*}}}\right|^{2}}{1+\gamma_{\mathrm{E}} \xi\left|h_{\mathrm{BS}}\right|^{2}\left|h_{\mathrm{SE}}\right|^{2}}, \\
& \gamma_{2 \mathrm{PRS}}=\frac{1+\gamma_{\mathrm{M}} \xi\left|h_{\mathrm{BR}_{k^{*}}}\right|^{2}\left|h_{\mathrm{R}_{k^{*}} \mathrm{D}}\right|^{2}}{1+\gamma_{\mathrm{E}} \xi\left|h_{\mathrm{BR}_{k^{*}}}\right|^{2}\left|h_{\mathrm{R}_{k^{*}} \mathrm{E}}\right|^{2}} .
\end{aligned}
$$


The CDF of $\gamma_{1 \mathrm{PRS}}$ is expressed as

$$
\begin{aligned}
& \mathbb{P}\left\{\gamma_{1 \text { PRS }}<x\right\} \\
& =1+\sum_{k=1}^{K} \sum_{v=0}^{l} \sum_{l=0}^{k\left(m_{\mathrm{SR}}-1\right)}\left(\begin{array}{l}
K \\
k
\end{array}\right)\left(\begin{array}{l}
v \\
l
\end{array}\right)(-1)^{k}(x-1)^{l-v}(\xi)^{v-l} \\
& \times\left(\gamma_{\mathrm{E}} x\right)^{v} \frac{\Gamma\left(v+m_{\mathrm{SE}}\right)}{\Gamma\left(m_{\mathrm{SE}}\right) \theta_{\mathrm{SE}}^{m_{\mathrm{SE}}}} \frac{w\left(l, k, m_{\mathrm{SR}}\right)}{\theta_{\mathrm{SR}}^{l} \gamma_{\mathrm{M}}^{l}} \\
& \times \frac{1}{\Gamma\left(m_{\mathrm{BS}}\right) \theta_{\mathrm{BS}}^{m_{\mathrm{BS}}}}\left(\frac{\theta_{\mathrm{SR}} \gamma_{\mathrm{M}} \theta_{\mathrm{SE}}}{k \gamma_{\mathrm{E}} x \theta_{\mathrm{SE}}+\theta_{\mathrm{SR}} \gamma_{\mathrm{M}}}\right)^{v+m_{\mathrm{SE}}} \\
& \times \mathbf{K}_{m_{\mathrm{BS}}-l+v}\left(2 \sqrt{\frac{k(x-1)}{\theta_{\mathrm{SR}} \theta_{\mathrm{BS}} \gamma_{\mathrm{M}} \xi}}\right) \\
& \times 2 \times\left(\frac{k(x-1) \theta_{\mathrm{BS}}}{\theta_{\mathrm{SR}} \gamma_{\mathrm{M}} \xi}\right)^{\frac{m_{\mathrm{BS}}-l+v}{2}} \text {. }
\end{aligned}
$$

The CDF of $\gamma_{2 \text { PRS }}$ is expressed as

$$
\begin{aligned}
& \mathbb{P}\left\{\gamma_{2 \mathrm{PRS}}<x\right\} \\
& =1-\sum_{i=0}^{m_{\mathrm{RD}}-1} \sum_{j=0}^{i}\left(\begin{array}{c}
i \\
j
\end{array}\right) \frac{1}{i !} \frac{(x-1)^{i-j}\left(\gamma_{\mathrm{E}} x\right)^{j}}{\gamma_{\mathrm{M}}^{i} \xi^{i-j} \theta_{\mathrm{RD}}^{i}} \\
& \times \frac{\Gamma\left(v+m_{\mathrm{RE}}\right)}{\Gamma\left(m_{\mathrm{RE}}\right) \theta_{\mathrm{RE}}^{m_{\mathrm{RE}}} \Gamma\left(m_{\mathrm{BR}}\right) \theta_{\mathrm{BR}}^{m_{\mathrm{BR}}}}\left(\frac{x \gamma_{\mathrm{E}}}{\gamma_{\mathrm{M}} \theta_{\mathrm{RD}}}+\frac{1}{\theta_{\mathrm{RE}}}\right)^{-\left(v+m_{\mathrm{RE}}\right)} \\
& \times \mathbf{K}_{m_{\mathrm{BR}}-i+j}\left(2 \sqrt{\frac{x-1}{\theta_{\mathrm{RD}} \theta_{\mathrm{BR} \gamma \mathrm{M} \xi}}}\right) \times 2 \\
& \times\left(\frac{\theta_{\mathrm{BR}}(x-1)}{\theta_{\mathrm{RD} \gamma \mathrm{M} \xi}}\right)^{\frac{m_{\mathrm{BR}-i+j}}{2}} .
\end{aligned}
$$

The SOP of the considered system in PRS scheme is formulated as follows

$$
F_{\gamma_{\mathrm{PRS}}}(\beta)=1-\left[\left(1-F_{\gamma_{\mathrm{IPRS}}}(\beta)\right)\left(1-F_{\gamma_{2 \mathrm{PRS}}}(\beta)\right)\right]
$$

After performing some mathematical manipulations, Eq. 22 can be achieved with the help of [30, Eq. (3.471.9)].

\section{Appendix B: proof of lemma 2}

In ORS scheme, the SNR of the first and second hop can be derived as

$$
\begin{aligned}
\gamma_{1 \mathrm{ORS}} & =\frac{1+\gamma_{\mathrm{M}} \xi\left|h_{\mathrm{BS}}\right|^{2}\left|h_{\mathrm{SR}_{k}}\right|^{2}}{1+\gamma_{\mathrm{E}} \xi\left|h_{\mathrm{BS}}\right|^{2}\left|h_{\mathrm{SE}}\right|^{2}} \\
\gamma_{2 \mathrm{ORS}} & =\frac{1+\gamma_{\mathrm{M}} \xi\left|h_{\mathrm{BR}_{k}}\right|^{2}\left|h_{\mathrm{R}_{k} \mathrm{D}}\right|^{2}}{1+\gamma_{\mathrm{E}} \xi\left|h_{\mathrm{BR}_{k}}\right|^{2}\left|h_{\mathrm{R}_{k} \mathrm{E}}\right|^{2}} .
\end{aligned}
$$

We denote that $Y_{o}=\left|h_{\mathrm{BS}}\right|^{2}$, and $Z_{o}=\left|h_{\mathrm{SE}}\right|^{2}$. The SOP of the considered system in the ORS scheme is calculated as follows

$$
\begin{aligned}
F_{\gamma_{\mathrm{ORS}}}(x)= & \int_{0}^{\infty} \int_{0}^{\infty}\left[1-\left(1-F_{\gamma_{1 \mathrm{ORS}} \mid Y_{o}, Z_{o}}(x)\right)\right. \\
& \left.\times\left(1-F_{\gamma_{2 \mathrm{ORS}}}(x)\right)\right]^{K} f_{Y_{o}}(y) f_{Z_{o}}(z) d y d z .
\end{aligned}
$$

Where in the first hop

$$
\begin{aligned}
& F_{\gamma_{1 \mathrm{ORS}} \mid Y_{o}, Z_{o}}(x) \\
& =\mathbb{P}\left\{\frac{1+\gamma_{\mathrm{M}} \xi\left|h_{\mathrm{BS}}\right|^{2}\left|h_{\mathrm{SR}_{k}}\right|^{2}}{1+\gamma_{\mathrm{E}} \xi\left|h_{\mathrm{BS}}\right|^{2}\left|h_{\mathrm{SE}}\right|^{2}}<x\right\} \\
& =\mathbb{P}\left\{\left|h_{\mathrm{SR}_{k}}\right|^{2}<\frac{\left(1+\gamma_{\mathrm{E}} \xi Y_{o} Z_{o}\right) x-1}{\gamma_{\mathrm{M}} \xi Y_{o}}\right\} \\
& =1-\exp \left(-\frac{\left[1+\gamma_{\mathrm{E}} \xi Y_{o} Z_{o}\right] x-1}{\gamma_{\mathrm{M}} \xi Y_{o} \theta_{\mathrm{SR}}}\right) \sum_{i=0}^{m_{\mathrm{SR}}-1} \frac{1}{i !} \\
& \times\left(\frac{\left[1+\gamma_{\mathrm{E}} \xi Y_{o} Z_{o}\right] x-1}{\gamma_{\mathrm{M}} \xi Y_{o} \theta_{\mathrm{SR}}}\right)^{i} .
\end{aligned}
$$

Where in the second hop

$$
\begin{aligned}
& F_{\gamma_{2 \mathrm{ORS}}}(x) \\
& =1-\sum_{u=0}^{m_{\mathrm{RD}}-1} \sum_{v=0}^{u}\left(\begin{array}{l}
u \\
v
\end{array}\right) \frac{1}{u !} \frac{(x-1)^{u-v}\left(\gamma_{\mathrm{E}} x\right)^{v}}{\gamma_{\mathrm{M}}^{u} \xi^{u-v} \theta_{\mathrm{RD}}^{u}} \\
& \times \frac{1}{\Gamma\left(m_{\mathrm{RE}}\right) \theta_{\mathrm{RE}}^{m_{\mathrm{RE}}}} \Gamma\left(v+m_{\mathrm{RE}}\right)\left(\frac{\gamma_{\mathrm{E}} x}{\gamma_{\mathrm{M}} \theta_{\mathrm{RD}}}+\frac{1}{\theta_{\mathrm{RE}}}\right)^{-\left(v+m_{\mathrm{RE}}\right)} \\
& \times 2 \times\left(\frac{[x-1] \theta_{\mathrm{BR}}}{\gamma_{\mathrm{M} \xi} \xi \theta_{\mathrm{RD}}}\right)^{\frac{v-u+m_{\mathrm{BR}}}{2}} \frac{1}{\Gamma\left(m_{\mathrm{BR}}\right) \theta_{\mathrm{BR}}^{m_{\mathrm{BR}}}} \mathbf{K}_{v-u+m_{\mathrm{BR}}} \\
& \times\left(2 \sqrt{\frac{x-1}{\gamma_{\mathrm{M} \xi} \theta_{\mathrm{RD}} \theta_{\mathrm{BR}}}}\right) .
\end{aligned}
$$

After performing some mathematical manipulations, Eq. 26 can be achieved with the help of [30, Eq. (3.471.9)].

\section{References}

1. Yuen C, Elkashlan M, Qian Y, Duong TQ, Shu L, Schmidt F (2015) Energy harvesting communications: part 1 [Guest Editorial]. IEEE Commun Mag 53(6):54-55

2. Yuen C, Elkashlan M, Qian Y, Duong TQ, Shu L, Schmidt F (2015) Energy harvesting communications: part 2 [Guest Editorial]. IEEE Commun Mag 53(6):54-55

3. Yuen C, Elkashlan M, Qian Y, Duong TQ, Shu L, Schmidt F (2015) Energy harvesting communications: part 3 [Guest Editorial]. IEEE Commun Mag 53(6):54-55

4. Zhou X, Zhang R, Ho CK (2013) Wireless information and power transfer: architecture design and rate-energy tradeoff. IEEE Trans Commun 61(11):4754-4767 
5. Nasir AA, Zhou X, Durrani S, Kennedy RA (2013) Relaying protocols for wireless energy harvesting and information processing. IEEE Trans Wireless Commun 12(7):3622-3636

6. Nasir AA, Zhou X, Durrani S, Kennedy RA (2015) Wirelesspowered relays in cooperative communications: time-switching relaying protocols and throughput analysis. IEEE Trans Commun 63(5):1607-1622

7. Michalopoulos DS, Suraweera HA, Schober R (2015) Relay selection for simultaneous information transmission and wireless energy transfer: a tradeoff perspective. IEEE J Sel Areas Commun 33(8):1-1

8. Mohammadi M, Chalise BK, Suraweera HA, Zhong C, Zheng G, Krikidis I (2016) Throughput analysis and optimization of wireless-powered multiple antenna full-duplex relay systems. IEEE Trans Commun 64(4):1769-1785

9. Zhong C, Zheng G, Zhang Z, Karagiannidis G (2015) Optimum wirelessly powered relaying. IEEE Signal Process Lett 22(10):1-1

10. Nguyen V-D, Duong TQ, Tuan HD, Shin O-S, Poor HV (2017) Spectral and energy efficiencies in full-duplex wireless information and power transfer. IEEE Trans Commun 65(5):2220-2233

11. Rankov B, Wittneben A (2007) Spectral efficient protocols for half-duplex fading relay channels. IEEE J Sel Areas Commun 25(2):379-389

12. Bao VNQ, Duong TQ, da Costa DB, Alexandropoulos GC, Nallanathan A (2013) Cognitive amplify-and-forward relaying with best relay selection in non-identical Rayleigh fading. IEEE Commun Lett 17(3):475-478

13. Chen G, Tian Z, Gong Y, Chambers J (2014) Decode-and-forward buffer-aided relay selection in cognitive relay networks. IEEE Trans Veh Technol 63(9):4723-4728

14. Hoang TM, Duong TQ, Vo NS, Kundu C (2017) Physical layer security in cooperative energy harvesting networks with a friendly jammer. IEEE Wireless Commun Lett PP(99):1-1

15. Fan L, Lei X, Yang N, Duong TQ, Karagiannidis GK (2017) Secrecy cooperative networks with outdated relay selection over correlated fading channels. IEEE Trans Veh Technol PP(99):1-1

16. Yang M, Guo D, Huang Y, Duong TQ, Zhang B (2016) Secure multiuser scheduling in downlink dual-hop regenerative relay networks over Nakagami- $m$ fading channels. IEEE Trans Wireless Commun 15(12):8009-8024

17. Fan L, Lei X, Yang N, Duong TQ, Karagiannidis GK (2016) Secure multiple amplify-and-forward relaying with cochannel interference. IEEE J Sel Topics Signal Process 10(8):1494-1505
18. Duong TQ, Hoang TM, Kundu C, Elkashlan M, Nallanathan A (2016) Optimal power allocation for multiuser secure communication in cooperative relaying networks. IEEE Wireless Commun Lett 5(5):516-519

19. Huang Y, Wang J, Zhong C, Duong TQ, Karagiannidis GK (2016) Secure transmission in cooperative relaying networks with multiple antennas. IEEE Trans Wireless Commun 15(10):6843-6856

20. Nguyen NP, Duong TQ, Ngo HQ, Hadzi-Velkov Z, Shu L (2016) Secure 5G wireless communications: a joint relay selection and wireless power transfer approach. IEEE Access 4:3349-3359

21. Fan L, Yang N, Duong TQ, Elkashlan M, Karagiannidis GK (2016) Exploiting direct links for physical layer security in multiuser multirelay networks. IEEE Trans Wireless Commun 15(6):3856-3867

22. Hoang TM, Duong TQ, Suraweera HA, Tellambura C, Poor HV (2015) Cooperative beamforming and user selection for improving the security of relay-aided systems. IEEE Trans Wireless Commun 63(12):5039-5051

23. Rodriguez LJ, Tran NH, Duong TQ, Le-Ngoc T, Elkashlan M, Shetty S (2015) Physical layer security in wireless cooperative relay networks: atate of the art and beyond. IEEE Commun Mag 53(12):32-39

24. Wang L, Kim KJ, Duong TQ, Elkashlan M, Poor HV (2015) Security enhancement of cooperative single carrier systems. IEEE Trans Inf Forensics Secur 10(1):90-103

25. Fan L, Lei X, Duong TQ, Elkashlan M, Karagiannidis GK (2014) Secure multiuser communications in multiple amplifyand-forward relay networks. IEEE Trans Commun 62(9):32993310

26. Wang L, Elkashlan M, Huang J, Tran NH, Duong TQ (2014) Secure transmission with optimal power allocation in untrusted relay networks. IEEE Wireless Commun Lett 3(3):289-292

27. Ng DWK, Lo ES, Schober R (2014) Robust beamforming for secure communication in systems with wireless information and power transfer. IEEE Trans Wireless Commun 13(8):4599-4615

28. Liu W, Zhou X, Durrani S, Popovski P (2016) Secure communication with a wireless-powered friendly jammer. IEEE Trans Wireless Commun 15(1):401-415

29. Zhang J, Nguyen N-P, Zhang J, Garcia-Palacios E, Le NP (2016) Impact of primary networks on the performance of energy harvesting cognitive radio networks. IET Commun 10(18):2559-2566

30. Gradshteyn IS, Ryzhik IM (2007) Table of integrals, series, and products, 7th edn. Academic Press, San Diego 